\title{
The Prevalence of Growth Hormone Deficiency and Celiac Disease in Short Children
}

\author{
Diletta Giovenale, MD; Cristina Meazza, PhD; Giuliana M. Cardinale, MD; Maddalena Sposito, MD; \\ Costanzo Mastrangelo, MD; Beatrice Messini, MD; Giuseppe Citro, MD; Maurizio Delvecchio, MD; \\ Salvatore Di Maio, MD; and Mauro Bozzola, MD
}

\begin{abstract}
Objectives: To assess the occurrence of growth hormone deficiency (GHD) in patients with celiac disease $(C D)$ referred for short stature.

Design: A retrospective, multi-center study. A total of 7066 children with short stature were referred to a number of centers for second-line evaluation over a 5-year period.All patients were screened for $\mathrm{CD}$ by antiendomysial antibodies (EMA) and antitissue transglutaminase IgA. Those with positive sera underwent intestinal biopsy. The EMA-negative patients and the EMA-positive ones who did not grow after I year of gluten-free diet underwent endocrinological investigation.
\end{abstract}

Results: Among the 7066 short children (age 2-14 years) evaluated, 650 (9.2\%) had GHD and 44 (0.63\%) had CD. An association of both CD and GHD was found in 16 short children (0.23\%); these children did not grow after I year of gluten-free diet and therefore $\mathrm{GH}$ treatment was started.

Conclusions: $\mathrm{GH}$ secretion should be evaluated in celiac patients showing no catch-up growth after an appropriate period on a gluten-free diet in spite of reversion to seronegativity for EMA.

Keywords: Antiendomysial antibodies; Celiac disease; Growth hormone deficiency; Prevalence; Short children

$\mathrm{C}$

eliac disease $(\mathrm{CD})$ is an immune-mediated enteropathy triggered by the ingestion of gluten in genetically susceptible individuals. The major CD-predisposing genes are located in the HLA region, namely the HLA-DQ2 and DQ8 genotypes, found in almost all CD patients and in $30 \%$ of the general population. ${ }^{1}$

$\mathrm{CD}$ is one of the most common lifelong disorders in Europe and in the United States. The prevalence of childhood CD has been reported to be between 1:230 and 1:106 in Italian school children.2,3 Furthermore, the prevalence of $\mathrm{CD}$ is greater in certain pediatric populations, including those with Down's syndrome, Turner's syndrome and William's syndrome. ${ }^{4-7}$ Among these associations, $\mathrm{CD}$ in Down's syndrome is the one that has been most studied, and in a multi-center Italian study, a prevalence of this disease association was found to be $4.6 \% .8$
The epidemiological pattern of CD has dramatically changed in the past few years as a result of the widespread use of highly sensitive and specific serological tests, especially antiendomysial (EMA) and antitissue transglutaminase (anti-tTG) antibodies. ${ }^{9}$ Despite the reported high prevalence, CD is commonly underdiagnosed in clinical practice probably because of its broad clinical spectrum in children and the presence of clinically silent forms. ${ }^{10}$ When CD manifests with atypical symptoms or is clinically silent, many patients remain undiagnosed and are exposed to the risk of long-term complications, such as osteoporosis, infertility and cancer. ${ }^{10}$ The CD form with classic symptoms is characterized by gastrointestinal manifestations, such as chronic diarrhea and abdominal distension, starting between 6 and 24 months of age and after the introduction of gluten in the diet. ${ }^{10}$ On the contrary, there may be a delayed onset of $\mathrm{CD}$ involving older children (5-7 years of age) who show unusual intestinal complaints (e.g., nausea, vomiting, recurrent abdominal pain) or extraintestinal manifestations, such as short stature, 
Table 1. Short children with celiac disease (CD), growth hormone deficiency (GHD) and the association of CD and GHD.

\begin{tabular}{lcccc}
\hline Pediatric outpatient centers & $\begin{array}{c}\text { Short stature } \\
\mathbf{n}\end{array}$ & $\begin{array}{c}\text { CD } \\
\mathbf{n}(\%)\end{array}$ & $\begin{array}{c}\text { GHD } \\
\mathbf{n}(\%)\end{array}$ & $\begin{array}{c}\text { CD and GHD } \\
\mathbf{n}(\%)\end{array}$ \\
\hline Pavia & 1066 & $10(0.94)$ & $210(19.7)$ & $4(0.38)$ \\
Foggia & 506 & $3(0.60)$ & $30(5.9)$ & $3(0.59)^{*}$ \\
Lecce & 609 & $3(0.49)$ & $61(10.0)$ & $1(0.16)$ \\
Foligno & 382 & $3(0.79)$ & $12(3.1)$ & $3(0.78)$ \\
Potenza & 106 & $4(3.77)$ & $12(11.3)$ & $1(0.94)$ \\
Perugia & 503 & $8(1.59)$ & $80(15.9)$ & $1(0.20)$ \\
Bari & 654 & $2(0.31)$ & $95(14.5)$ & $1(0.15)$ \\
Napoli & 3240 & $11(0.34)$ & $150(4.6)$ & $2(0.06)$ \\
\hline Total & 7066 & $44(0.63)$ & $650(9.2)$ & $16(0.23)$ \\
\hline
\end{tabular}

* One is Down syndrome and one is Turner syndrome.

pubertal delay, iron deficiency and dental enamel defects. ${ }^{10,11}$ In some cases short stature may be the only symptom, and the diagnosis of these cases is often difficult. ${ }^{12-14}$ Some authors have described patients with $\mathrm{CD}$ and short stature, normal growth hormone release and low somatomedin levels. ${ }^{15}$ Among our endocrinological patients with growth failure, we recently found three cases of $\mathrm{CD}$ associated with growth hormone deficiency (GHD). ${ }^{16}$

The aim of this study was to further evaluate the relationship between GHD and $\mathrm{CD}$, investigating the prevalence of $\mathrm{CD}$ and GHD in children screened for short stature in eight pediatric endocrinological centers of different Italian regions.

\section{Patients and Methods}

In this retrospective, multi-center study, a total of 7066 children aged 2 years to 14 years (4240 males, 2826 females) were screened for short stature during a period of 5 years of outpatient activity in Pavia, Naples, Foggia, Lecce, Foligno, Potenza, Perugia and Bari, Italy. In order to exclude other causes of short stature, thyroid and adrenal functions were investigated by evaluating serum free thyroxine (FT4) and thyroid stimulating hormone (TSH), and morning and evening serum cortisol concentrations, respectively. All subjects were then screened for CD, even if they were asymptomatic. Serum was assayed for EMA and anti-tTG IgA. Children positive twice for EMA and anti-tTG IgA underwent an endoscopic examination of the upper gastrointestinal tract with at least four biopsies of the distal duodenal mucosa. Villous atrophy (Marsh type 3), infiltrative changes (Marsh type 1) and crypt hyperplasia (Marsh type 2) were considered characteristic histopathologic features of CD. ${ }^{17}$

Children classified as "slightly positive" for EMA were followed. During the follow-up the children did not start a gluten-free diet, and after 2 to 3 months of a diet rich in gluten, EMA was reassayed. If the EMA results were again positive, the patient underwent endoscopic examination for a confirmatory final diagnosis.
In short children with growth failure and seronegativity for EMA, growth hormone secretion was evaluated after at least two pharmacological stimuli. A diagnosis of GHD was established when serum growth hormone peaks were lower than $10 \mathrm{ng} / \mathrm{ml}$ in the presence of short stature, reduced growth velocity and delayed bone age. ${ }^{18}$ All CD patients began a gluten-free diet and were followed up every 6 months. The CD children showing no catch-up growth (growth velocity $\pm \mathrm{SD}$, $-2.45 \pm 1.42$ ) after a 12-month gluten-free diet period were further investigated by evaluating growth hormone secretion, as is done for slowly growing short children.

Basal auxological data (mean \pm standard deviation) of patients presenting both $\mathrm{CD}$ and GHD at time of $\mathrm{CD}$ diagnosis were height, $-2.17 \pm 0.44$; growth velocity, $-0.74 \pm 0.95$; bone age (years) $5.65 \pm 4.26$; and IGF-I $(\mathrm{ng} / \mathrm{ml}), 130.7 \pm 78.8$.

Serum growth hormone, FT4 and TSH concentrations were measured using commercial time-resolved fluoroimmunoassays. EMA IgA was detected by indirect immunofluorescence on sections from the distal portion of monkey esophagus as an antigenic substrate. Serum anti-tTG IgA was determined using a commercially available enzyme-linked immunosorbent assay (ELISA) kit (Eu-tTG IgA, Eurospital, Trieste, Italy). All centers participating in the study used the same ELISA kit.

\section{Results}

As shown in table 1, we studied children with short stature admitted to eight pediatric outpatient centers located in different regions of Italy. The screening of 7066 short stature children by EMA antibody and anti-tTG IgA determinations followed by intestinal biopsy in positive cases led to the diagnosis of CD in $44(0.63 \%)$ subjects who, however, did not show gastrointestinal symptoms. Out of the EMA-negative children, we found $650(9.2 \%)$ subjects with GHD.

An association of GHD and CD was found in 16 children $(0.23 \%)$. Among this group of patients, one subject with Down's syndrome and one with Turner's syndrome were observed. Children with both GHD and CD did not grow, in 
spite of the gluten-free diet and reversion to seronegativity for EMA, and started growth hormone therapy at the dose of 0.25 $\mathrm{mg} / \mathrm{kg}$ in six divided injections per week. The growth velocity of these children improved after the first year of growth hormone treatment, but a longer follow-up is needed to evaluate the true effectiveness of the therapy.

\section{Discussion}

$\mathrm{CD}$ is a chronic inflammatory condition associated with small intestinal injury caused by intolerance of gluten in genetically susceptible individuals. ${ }^{1}$

In the diagnostic approach to short stature children, $\mathrm{CD}$ has to be excluded even if the subjects do not show gastrointestinal symptoms. CD is often underdiagnosed in clinical practice because clinically overt cases account for only a minority of $\mathrm{CD}$ patients. Fortunately, in the last few years, the use of highly sensitive and specific serological tests, antiendomysial and anti-tTG antibodies has led to the identification of atypical forms of $\mathrm{CD}$, such as those in which short stature may be the only symptom. ${ }^{12-14}$ This increased number of diagnosed $C D$ cases has dramatically changed the epidemiology of the disease.

The pathogenesis of $\mathrm{CD}$-associated short stature is still unclear. Growth retardation has traditionally been attributed to generalized or selective malnutrition. Indeed, after starting the gluten-free diet, a significant increase in height velocity is often noticed, especially during the first year of gluten restriction. However, the catch-up growth is not always complete, probably due to the marked acceleration in bone maturation. Furthermore, children in which a catch-up growth, at least incomplete, is not reached could present some endocrinological abnormalities, such as GHD, as shown in this study. Therefore, in children with growth failure that does not improve after starting a gluten-free diet, careful endocrinological evaluation should be considered as GHD is another well known cause of short stature. It is characterized by reduced growth velocity, delayed bone age and blunted growth hormone secretion after pharmacological stimuli. ${ }^{18}$

We recently described three short children with $\mathrm{CD}$ and GHD, who increased their growth rate only after gluten-free diet associated with growth hormone therapy, suggesting a possible association between these two diseases. ${ }^{16}$

In the study presented here, in unselected cases admitted for short stature, the prevalence of $\mathrm{CD}$ varies from $0.31 \%$ to $3.77 \%$, with a mean value of $0.63 \%$, which is very similar to the prevalence described in Italian children. ${ }^{3}$ Some authors $12,19,20$ have reported higher prevalence of CD in short children. However, these reports are controversial because either the population with short stature has often not been clearly defined on auxological and endocrinological bases, or the diagnosis of $\mathrm{CD}$ was not according to ESPGAN criteria. In the same group of patients the prevalence of GHD was calculated to be $9.2 \%$, with some centers showing higher values of about $15 \%$ to $20 \%$. The epidemiological value reported in the literature for GHD in childhood is about $0.02 \%$ to $0.03 \%,{ }^{21,22}$ which is much lower as compared to our results. However, we calculated the prevalence of GHD in a population of short children, and the outpatient departments participating in the study are second-line or third-line level centers that receive children who have already been screened by other physicians. The prevalence value of GHD that our present study determined is comparable to that reported by Kaplowitz et $\mathrm{al}^{23}$ who determined GHD values in a population of short children with height $\geq 3 \mathrm{SD}$ below the mean.

Out of 7066 children with short stature, we found $16(0.23 \%)$ subjects having both GHD and CD. Currently, they are on both a gluten-free diet and growth hormone treatment and, although a prolonged follow-up is needed, it seems that their growth velocity has improved. These results further support our previous observations on a restricted number of patients and confirm that an association could exist between $\mathrm{CD}$ and GHD in unselected short children.

Interestingly, among the patients with both CD and GHD we found one subject with Down's syndrome and one with Turner's syndrome. It has been reported previously that there is an association between some genetic disorders and CD. ${ }^{4-7}$ However, our 2 patients are too few to confirm this observation. In Down's syndrome, $\mathrm{CD}$ is not detectable on the basis of clinical findings alone and is underdetected. There is, however, good evidence that in Down's syndrome, some subjects, who initially have a negative serological test, may subsequently develop a positive test on repeat testing over a period of years. Testing is, therefore, recommended for asymptomatic children who have conditions associated with $\mathrm{CD}$, such as Turner's, Down's and William's syndrome. ${ }^{4-7}$

In conclusion, growth hormone secretion should be evaluated in $\mathrm{CD}$ patients showing no catch-up growth after a gluten-free diet, and in patients with both CD and GHD, replacement growth hormone therapy should be considered only after a gluten-free diet period.

\section{References}

1. Fasano A. Clinical presentation of celiac disease in the pediatric population. Gastroenterology 2005;128:S68-S73.

2. Catassi C, Ratsch IM, Fabiani E, Ricci S, Bordicchia F, Pierdomenico R, Giorgi PL. High prevalence of undiagnosed coeliac disease in 5280 Italian students screened by antigliadin antibodies. Acta Paediatr 1995;84:672-676.

3. Tommasini A, Not T, Kiren V, Baldas V, Santon D, Trevisiol C, Berti I, Neri E, Gerarduzzi T, Bruno I, Lenhardt A, Zamuner E, Spano A, Crovella S, Martellossi S, Torre G, Sblattero D, Marzari R, Bradbury A, Tamburlini G, Ventura A. Mass screening for coeliac disease using antihuman transglutaminase antibody assay. Arch Dis Child 2004;89:512-515.

4. Book L, Hart A, Black J, Feolo M, Zone JJ, Neuhausen SL. Prevalence and clinical characteristics of celiac disease in Downs syndrome in a US study. Am J Med Genet 2001;98:70-74. 
5. Mackey J, Treem WR, Worley G, Boney A, Hart P, Kishnani PS. Frequency of celiac disease in individuals with Down syndrome in the United States. Clin Pediatr (Phila) 2001:40:249-252.

6. Bonamico M, Pasquino AM, Mariani P, Danesi HM, Culasso F, Mazzanti L, Petri A, Bona G; Italian Society Of Pediatric Gastroenterology Hepatology (SIGEP); Italian Study Group for Turner Syndrom (ISGTS). Prevalence and clinical picture of celiac disease in Turner syndrome. J Clin Endocrinol Metab 2002;87:5495-5498.

7. Giannotti A, Tiberio G, Castro M, Virgilii F, Colistro F, Ferretti F, Digilio MC, Gambarara M, Dallapiccola B. Coeliac disease in Williams syndrome. J Med Genet 2001;38:767-768.

8. Bonamico M, Mariani P, Danesi HM, Crisogianni M, Failla P, Gemme G, Quartino AR, Giannotti A, Castro M, Balli F, Lecora M, Andria G, Guariso G, Gabrielli O, Catassi C, Lazzari R, Balocco NA, De Virgiliis S, Culasso F, Romano C; SIGEP (Italian Society of Pediatric Gastroenterology and Hepatology) and Medical Genetic Group. Prevalence and clinical picture of celiac disease in Italian down syndrome patients: a multicenter study. J Pediatr Gastroenterol Nutr 2001;33:139-143.

9. Green PH, Jabri B. Coeliac disease. Lancet 2003;362:383-391.

10. Fasano A, Catassi C. Current approaches to diagnosis and treatment of celiac disease: an evolving spectrum. Gastroenterology 2001;120:636-651.

11. Visakorpi JK, Maki M. Changing clinical features of coeliac disease. Acta Paediatr Suppl 1994;83:10-13.

12. Tumer L, Hasanoglu A, Aybay C. Endomysium antibodies in the diagnosis of celiac disease in short-statured children with no gastrointestinal symptoms. Pediatr Int 2001;43:71-73.

13. Cacciari E, Salardi S, Volta U, Biasco G, Lazzari R, Corazza GR, Feliciani M, Cicognani A, Partesotti S, Azzaroni D. Can antigliadin antibody detect symptomless coeliac disease in children with short stature? Lancet 1985;1:1469-1471.

14. Groll A, Candy DC, Preece MA, Tanner JM, Harries JT. Short stature as the primary manifestation of coeliac disease. Lancet 1980;2:1097-1099.

15. Day G, Evans K, Wharton B. Abnormalities of insulin and growth hormone secretion in children with coeliac disease. Arch Dis Child 1973;48:41-46.

16. Bozzola M, Giovenale D, Bozzola E, Meazza C, Martinetti M, Tinelli C, Corazza GR. Growth hormone deficiency and coeliac disease: an unusual association? Clin Endocrinol (Oxf) 2005;62:372-375.

17. Hill ID, Dirks MH, Liptak GS, Colletti RB, Fasano A, Guandalini S, Hoffenberg EJ, Horvath K, Murray JA, Pivor M, Seidman EG; North American Society for Pediatric Gastroenterology, Hepatology and Nutrition. Guideline for the diagnosis and treatment of celiac disease in children: recommendations of the North American Society for Pediatric Gastroenterology, Hepatology and Nutrition. J Pediatr Gastroenterol Nutr 2005;40:1-19.

18. Growth Hormone Research Society. Consensus guidelines for the diagnosis and treatment of growth hormone $(\mathrm{GH})$ deficiency in childhood and adolescence: summary statement of the GH Research Society. GH Research Society. J Clin Endocrinol Metab 2000;85:3990-3993.

19. Knudtzon J, Fluge G, Aksnes L. Routine measurements of gluten antibodies in children of short stature. J Pediatr Gastroenterol Nutr 1991;12:190-194.

20. Cacciari E, Salardi S, Lazzari R, Cicognani A, Collina A, Pirazzoli P, Tassoni P, Biasco G, Corazza GR, Cassio A. Short stature and celiac disease: a relationship to consider even in patients with no gastrointestinal tract symptoms. J Pediatr 1983;103:708-711.

21. Lindsay R, Feldkamp M, Harris D, Robertson J, Rallison M. Utah Growth Study: growth standards and the prevalence of growth hormone deficiency. J Pediatr 1994;125:29-35.
22. Thomas M, Massa G, Craen M, de Zegher F, Bourguignon JP, Heinrichs C, De Schepper J, Du Caju M, Thiry-Counson G, Maes M. Prevalence and demographic features of childhood growth hormone deficiency in Belgium during the period 1986-2001. Eur J Endocrinol 2004;151:67-72.

23. Kaplowitz P, Webb J. Diagnostic evaluation of short children with height 3 SD or more below the mean. Clin Pediatr (Phila) 1994;33:530-535.

\section{Author Affiliations}

Diletta Giovenale, MD, Dipartimento di Scienze

Pediatriche, Università degli Studi di Pavia, IRCCS

Policlinico San Matteo, Pavia, Italy

Cristina Meazza, PhD, Dipartimento di Scienze Pediatriche, Università degli Studi di Pavia, IRCCS Policlinico San Matteo, Pavia, Italy

Giuliana M. Cardinale, MD, Divisione di Pediatria, Ospedale "F. Ferrari", Casatano, Lecce, Italy

Maddalena Sposito, MD, Clinica Pediatrica Monteluce, Perugia, Italy

Costanzo Mastrangelo, MD, U.O. Pediatria, Ospedali Riuniti, Foggia, Italy

Beatrice Messini, MD, U.O. Pediatrica, Ospedale di Foligno, Foligno, Italy

Giuseppe Citro, MD, U.O. Endocrinologica, Ospedale San Carlo, Potenza, Italy

Maurizio Delvecchio, MD, Dipartimento di Biomedicina dell'età evolutiva, Università degli studi di Bari, Bari, Italy

Salvatore Di Maio, MD, Dipartimento di Pediatria e Riabilitazione, Azienda Ospedaliera Pediatrica "Santobono-Pausilipon”, Napoli, Italy

Mauro Bozzola, MD, Dipartimento di Scienze Pediatriche, Università degli Studi di Pavia, IRCCS Policlinico San Matteo, Pavia, Italy 